\title{
IL «PLATONE» DI PAUL FRIEDLÄNDER: LA SUA IMPORTANZA E LA SUA PORTATA STORICO-ERMENEUTICA
}

\section{La figura di Paul Friedländer come filologo e storico}

Paul Friedländer è nato a Berlino nel 1882 e si è formato sotto la guida di professori di filologia classica di grande valore e fama quali Hermann Usener e Ulrich von Wilamowitz Moellendorff.

Gli anni per lui più fecondi e più ricchi - sia nelle sue ricerche sia nel suo magistero - sono stati quelli trascorsi all'Università di Marburg dal 1920 in qualità di professore ordinario di filologia classica.

A partire dal 1932 passò all'Università di Halle, dove poté restare solo per breve tempo. Infatti, in quanto era di origini semitiche, in conseguenza delle leggi emanate dal nazismo contro gli ebrei, già nel 1935 gli veniva tolta la cattedra e veniva rimosso dall'insegnamento.

Dopo un breve periodo di tempo trascorso a Roma come bibliotecario della Gregoriana e della Vaticana, emigrò negli Stati Uniti, dove insegnò dapprima all'Università di Baltimora come lettore e successivamente all'Università di Los Angeles come professore di filologia classica. Morì nel 1968.

Friedländer si formò soprattutto su basi filologiche e archeologiche nell'ambito dell'antichità, ma si occupò anche di letteratura moderna e scrisse - tra l'altro - un'opera sul Faust di Goethe (Rbytbmen und Landscbaften im Zweiten 
Teil des Faust, Weimar 1953). Si occupò - tra l'altro - anche di arti figurative, con lavori che rimangono nell'ambito della loro tematica ancora oggi un punto di riferimento (Johannes von Gaza und Paulus Silentiarius. Kunstbeschreibungen Justinianischer Zeit, Leipzig-Berlin 1912 e Spätantiker Gemäldezyklus in Gaza, in «Studi e testi della Biblioteca Vaticana», LXXXIX, 1939 (opere riedite anche insieme, Hildesheim 1969).

Il punto focale degli interessi di Friedländer fu proprio Platone, dagli inizi fino al termine della sua vita. Incominciò con due volumi sull'Alcibiade maggiore (Der grosse Alkibiades. Ein Weg zu Plato, Bonn 1921-1923), pubblicò anche vari articoli, ma la figura del grande studioso e interprete emerse soprattutto nella imponente opera generale Platone, dapprima edita in due volumi (1928-1930), successivamente in tre volumi (1957-1960), con la stesura definitiva della terza edizione sempre in tre volumi, del 1964.

Qui viene presentata in modo unitario la traduzione dei tre volumi dell'edizione definitiva (fatta con grande acribia e competenza da Andrea Le Moli): il primo volume viene presentato come «Libro primo», mentre gli altri due volu$\mathrm{mi}$ - che solo per ragioni editoriali erano divisi, ma sono fra di loro strettamente legati - vengono presentati come «Libro secondo». Nelle testatine, accanto alla paginazione di questa edizione italiana viene indicata anche la corrispettiva paginazione della terza edizione dell'originale tedesco.

L'opera si impone come un vero e proprio classico nell'ambito della letteratura critica su Platone, e per molte ragioni si impone come un punto di riferimento per chiunque voglia comprendere Platone e la storia delle interpretazioni del pensiero del grande filosofo.

Si potrebbe addirittura dire che - sotto certi aspetti - l'opera può essere considerata la più bella e accattivante nell'ambito del paradigma ermeneutico tradizionale, e in particolare una delle più rappresentative e significative sia nei suoi pregi, sia anche nei suoi difetti. 
I pregi di quest'opera stanno soprattutto in due dei suoi punti-chiave, di cui parleremo in maniera dettagliata più avanti, e che qui anticipiamo.

In primo luogo, Friedländer ha compreso ed espresso meglio di tutti il nesso ideale strutturale che sussiste fra Socrate e Platone, e ha spiegato in maniera mirabile in che senso la figura di Socrate segni il "destino" di Platone. Ciò che in Socrate era prevalentemente "domanda" in Platone diventa anche "risposta"; ciò che in Socrate era soprattutto un "vissuto" in Platone diventa anche "dottrina".

In secondo luogo, Friedländer ha compreso e spiegato meglio di tutti la ragione per cui la forma letteraria del "dialogo" si impone in Platone come una "necessità", in quanto diventa una forma esemplare di "libro" che nello stesso tempo è un "non-libro", ossia sotto certi aspetti ricupera e supera il libro medesimo. Lo studioso scrive: «Così egli solo, anche se i riflessi del dialogo socratico dovettero prima di lui prendere carattere letterario, può chiamarsi creatore del dialogo filosofico come di un'opera d'arte alla pari con la grande tragedia e la grande commedia» (infra, p. 185).

I punti deboli dell'opera di Friedländer dal punto di vista interpretativo sono soprattutto i seguenti.

In primo luogo, lo studioso tratta le Idee in maniera riduttiva al massimo grado. Infatti, nega che in Platone ci sia una "dottrina delle Idee", e afferma che tale "dottrina" è una creazione degli interpreti. Tuttavia, nello stesso tempo Friedländer ammette che Platone «ha creato un mondo metafisico» e che il compito che ha assunto è «quello di farlo vedere agli altri con i suoi occhi» (cfr. infra, pp. 18, 197). Inoltre, è costretto ad ammettere che, sia pure nella tarda Lettera VII (322 D), Platone parla di «sapienza delle Idee»

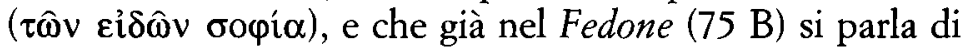
«scienza dell'uguale in sé», ossia di «scienza dell'Idea del-

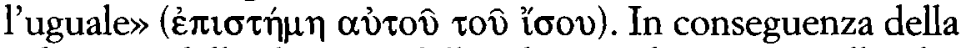
riduzione della dottrina delle Idee in Platone, Friedländer sottovaluta tutti i passi dei dialoghi in cui si fa riferimento alla dottrina medesima. 
In secondo luogo, dà alla problematica dell'arrbeton, ossia all' "indicibile", un significato che eccede la giusta misura. Infatti, egli ritiene che Platone riferisse quel termine addirittura a tutta quanta la sfera del mondo delle Idee, e non solo alla sfera dei Principi primi e supremi e in particolare al Bene. Inoltre, nei testi platonici - come vedremo l'arrbeton è riferito al Bene non in senso assoluto ma relativo: il Bene non è "indicibile" in sé e per sé, ma non è conoscibile e dicibile "come le altre cose", e dunque è conoscibile e dicibile seguendo una diversa via rispetto alla conoscenza delle altre cose.

Queste prese di posizione "riduttivistiche" da parte di Friedländer si spiegano e si giustificano soprattutto come presa di posizione contro una diffusa tendenza - soprattuto in Germania, e in particolare a Marburg con i Neokantiani alla testa - di interpretare le Idee secondo canoni della filosofia moderna. Ma questo difetto comporta anche un pregio, ossia quello di mettere in guardia l'interprete dal cercare di fare emergere in Platone quelle convinzioni teoretiche e sistematiche che non sono di Platone ma appunto dell'interprete.

Si deve tenere ben presente anche il fatto che Friedländer si è formato - come abbiamo sopra detto - come filologo e letterato, che alla filosofia è approdato studiando Platone, e che quindi proprio in conseguenza di questa sua formazione filologica egli tende a ridurre la specifica portata speculativa e lo spessore teoretico di certe affermazioni e di certe prese di posizione del filosofo.

Ben si può dire che sia corretto il tentativo di cercare di leggere e intendere Platone ponendosi al di qua dei modi in cui lo hanno ripensato molti Platonici e i Neoplatonici. Tuttavia va subito precisato che una lettura di Platone che cerca di non chiuderlo in un preciso sistema categoriale, non può e non deve essere comunque una lettura di carattere "precategoriale" in senso totale. In effetti, c'è in Platone un preciso tracciato categoriale - nascente nei primi dialoghi e via via sviluppantesi nel corso dei dialoghi della maturità e della vecchiaia - che come tale va riconosciuto e chiarito. 


\section{La corrente filosofica cui Friedländer si ispira}

Un certo clima culturale e una certa tendenza filosofica cui Friedländer si ispira sono ben identificabili.

In primo luogo bisogna tenere ben presente il fatto che, proprio nel giro di tempo in cui nasceva e veniva pubblicato il Platone di Friedländer, vedevano la luce opere di portata epocale, scritte da uomini che il nostro autore ben conosceva e frequentava. Nel 1923 usciva di Ernst Cassirer la Philosopbie der Symbolischen Formen. Nello stesso anno Werner Jaeger pubblicava il suo Aristoteles. Nel 1927 di Martin Heidegger usciva Sein und Zeit. Nel 1928 Julius Stenzel pubblicava il suo Platon der Erzieber e Werner Jaeger (che con Stenzel rilanciava il programma del "terzo umanesimo") dava alle stampe il saggio Platon im Aufbau der griechischen Bildung, contenente il progetto eseguito nella grandiosa opera Paideia (il cui volume I doveva uscire nel 1933 e i volumi II e III prima in lingua inglese nel 1953 e 1944 e poi nel 1944 e nel 1947 in tedesco; il lettore interessato potrà vedere quest'opera raccolta in volume unico, da noi edita in questa stessa collana nel 2003).

Ma la tendenza filosofica che Friedländer segue - pur risentendo influssi delle opere degli autori citati - sembra essere più vicina a quella tracciata dal "George-Kreis", ispirata a un particolare concetto di "Lebensphilosophie", o "filosofia della vita", che nell'opera di ciascuno dei grandi autori vedeva espressa la forma della vita dell'autore medesimo.

Gli autori e gli scritti platonici ispirati al "George-Kreis" sono: Heinrich Friedemann, Platon. Seine Gestalt, Berlin 1914; Heinrich Barth, Die Seele in der Pbilosopbie Platons, Tübingen 1921 (si tratta del fratello del teologo Karl Barth); Karl Reinhardt, Platons Mythen, Bonn 1927; Kurt Singer, Platon, der Gründer, München 1927.

$\mathrm{Ma}$ Friedländer si colloca ben al di sopra di questi autori e delle loro opere, in quanto imprime al programma del "George-Kreis" un differente spessore, e lo trasporta su un piano superiore, sia dal punto di vista concettuale che da quello storico-ermeneutico. 
Lo stesso sottotitolo dato da Friedländer alla sua opera, ossia Verità dell'essere e realtà del vivere - inteso come equivalente e sostituibile con Idea ed esistenza -, è assai significativo, in quanto indica quel particolare concetto di Lebensphilosophie, in cui si configura la circolarità dinamico-relazionale fra "essere" e "vivere": la verità dell'essere viene cercata con la realtà della vita, o, per converso, la realtà del vivere è continua ricerca della verità dell'essere.

Scrive Friedländer, dialogando con la filosofia esistenzialistica di Jaspers: «E così davvero il dialogo platonico è "esistenziale" in un senso più radicale di quel così ammirevole "chiarimento" dell'esistenza dello Jaspers. Perché quel che Jaspers compie è descrizione, analisi, sistemazione dell'umana esistenza, qua e là con una parola svegliando alla pura esistenza. Il Fedone, il Simposio e altri dialoghi sono drammi in cui questa umana esistenza si rappresenta. Ma essi non sono, o non sono soltanto, opere d'arte, di fronte alle quali si resta ammirati, ma sono una vita filosofica che invita il lettore a vivere insieme, a entrare nel dialogo, a contrastare e consentire. Essi non filosofeggiano sull'esistenza, ma sono - non dappertutto ma in gran parte - esistenza. Oppure, per non usare - e abusare - sempre dello stesso termine: sono la realtà del vivere, mentre cercano la verità dell'essere» (infra, p. 267).

In effetti, eccetto Socrate, nessun altro filosofo greco si presta a essere inteso con questo criterio meglio di Platone.

\section{Friedländer come maestro di Hans-Georg Gadamer}

Prima di entrare nel vivo dell'interpretazione di Platone fornita dal nostro studioso, c'è ancora un rilievo preliminare da mettere bene in evidenza, in quanto viene per lo più sottaciuto, se non addirittura ignorato da non pochi studiosi.

La formazione metodologica e uno dei primi germi da cui è nata l'ermeneutica di Hans-Georg Gadamer dipendono proprio da Paul Friedländer.

Per lo più Gadamer viene considerato un heideggeriano, e non viene riconosciuto il fatto che senza il pensiero classi- 
co e Platone le sue opere, e in particolare Verità e metodo (che il lettore interessato potrà trovare con testo tedesco a fronte in questa collana), risultano incomprensibili. Inoltre, non vengono spiegati il fatto e la ragione per cui Gadamer ha costruito il suo pensiero proprio sulle basi di carattere filologico acquisite alla scuola di Friedländer (fra i pochissi$\mathrm{mi}$ autori che mettono in evidenza questo punto va segnalato Jean Grondin con la sua grandiosa opera Gadamer. Una biografia, uscita in questa stessa collana nel 2004, curata da G.B. Demarta).

Da Heidegger (ben più che da Hartmann, con cui aveva sostenuto la sua tesi di laurea) Gadamer trasse l'ispirazione filosofica. In Maestri e compagni nel cammino del pensiero (Brescia 1980, p. 29) scrive: «Dall'incontro con Heidegger, per il quale ero andato un semestre a Friburgo e che ora seguivo a Marburgo, ebbi la prima conferma che quello che avevo praticato, con passione giocosa e solo con mezza soddisfazione, negli astratti esercizi intellettuali guidati da Nicolai Hartmann, non era ancora quello che io cercavo come filosofia. Lo stesso Nicolai Hartmann si era chiaramente accorto che seguivo il suo pensiero in maniera piuttosto imitativa e segretamente resistevo alla sua impostazione storica, per cui quando in Heidegger trovai confermata questa mia resistenza - soprattutto nei confronti dell'immersione interpretativa nella peculiarità storica dei testi filosofici - venne meno la fedeltà dell'antico scolaro verso Hartmann e mi incamminai sulle vie di Heidegger. Ma ogni nuovo inizio ha anche le sue difficoltà; rivelatasi insufficiente la mia preparazione e trovatomi troppo inferiore alle nuove esigenze dovetti sopportare una seconda volta la delusione del principiante. Furono anni di profondi dubbi sulle mie capacità scientifiche, ma insieme anche anni nei quali, finalmente, incominciai a lavorare sul serio. Divenni filologo classico, sotto la guida amichevole di Paul Friedländer».

In Verità e metodo 2 (a cura di R. Dottori, Bompiani 1995, p. 462) precisa: «Che cos'è che mi attirava così verso Heidegger? Naturalmente allora non lo sapevo. Oggi la cosa mi si mostra così. In lui le forme concettutali della tradizio- 
ne filosofica diventavano vive, perché le si comprendeva come risposte a reali domande. La messa a nudo della storia della loro motivazione conferiva a tali questioni qualcosa di ineluttabile».

Dunque, proprio per evitare di lavorare nel vago nel cercare di mettere in atto l'impulso che aveva ricevuto da Heidegger, Gadamer divenne filologo. In Verità e metodo 2 (p. 464) ribadisce: «Così iniziai un nuovo studio pianificato della filologia classica (sotto la guida di Paul Friedländer, in cui, oltre ai filosofi greci, mi attirò il poeta allora illuminato da un Hölderlin ridiventato accessibile, Pindaro), e dalla retorica, di cui capii allora la sua funzione complementare per la filosofia, che mi ha accompagnato fin nella elaborazione della mia ermeneutica filosofica». E nei Maestri e compagni (p. 35) precisa: «Con Paul Friedländer, che allora stava preparando la sua grande opera su Platone, leggevamo molto il grande filosofo greco».

Platone si impose in tal modo al centro degli studi di Gadamer, e fu l'autore in cui egli penetrò più a fondo. In Verità e metodo 2 (p. 486) dice: «Così, più che $i$ grandi pensatori dell'Idealismo tedesco, banno impresso un segno su di me $i$ dialoghi platonici, che mi hanno sempre accompagnato. Essi sono una compagnia del tutto particolare, per quanto noi, ammaestrati da Nietzsche e da Heidegger, possiamo avvertire come un limite la pregiudiziale della concettualità greca, che regna da Aristotele fino a Hegel e alla moderna logica, come un limite al di là del quale le nostre domande restano senza risposta, e le nostre intenzioni insoddisfatte. Bisogna però riconoscere che l'arte platonica del dialogo ha prevenuto anche questa parvenza di superiorità, che noi crediamo di possedere, quali eredi della tradizione greco-cristiana. Certamente è stato proprio lui, con la dottrina delle idee e la dialettica delle idee, con la matematizzazione della fisica e l'intellettualizzazione di quel che chiamiamo etica, a porre le basi della concettualità metafisica della nostra tradizione. Ma egli ha al tempo stesso limitato mimeticamente tutte le sue asserzioni, e come Socrate sapeva fare con la sua solita ironia con i suoi partner del dialogo, così Platone deruba 
anche il lettore della sua propria parvenza di superiorità tramite l'arte della poesia e del dialogo. Filosofare con Platone, non criticare Platone: questo è il compito. Criticare Platone forse è altrettanto ingenuo che rimproverare Sofocle di non essere Shakespeare. Questo suona paradossale, ma solo per colui che è cieco rispetto alla rilevanza dell'immaginazione poetica. - Certo bisogna prima imparare a leggere mimeticamente Platone. Nel nostro secolo è stato fatto qualcosa del genere, in particolare da Paul Friedländer [...]».

Proprio in riferimento a questo testo, e all'ammirazione più volte espressa da Gadamer per Hegel - che viene considerato $\mathrm{da}$ lui addirittura «il più grande successore dei Greci» - in una intervista che gli abbiamo fatto (per il Sole 24 Ore, domenica 17 settembre 2000), gli abbiamo domandato se amasse Platone più dello stesso Hegel. E la sua risposta è stata la seguente: «Certamente! Anzi, Le posso dire che amo Platone non solo più di Hegel, ma anche più dello stesso Heidegger».

E il Platone che Gadamer ripensa e fa proprio ha fortissime tangenze con il Platone di Friedländer.

Proprio il "dialogo", che secondo Friedländer è una "necessità" per Platone - nel modo in cui vedremo -, diventa per Gadamer l'asse portante della sua ermeneutica.

La preminenza metodologica della "domanda" nel discorso filosofico è determinante a tutti gli effetti. Il saper "porre domande" è più importante, e nello stesso tempo più difficile che "dare risposte". La domanda, infatti, predetermina l'area in cui si colloca la risposta, nonché la dinamica e l'articolazione della medesima, e quindi predetermina il procedimento del discorso in generale (come Platone stesso dimostra nel Protagora e nel Gorgia). Questo Gadamer mette bene in evidenza, ma già Friedländer lo ha compreso e detto.

Sorprendente è, poi, la sostanziale tangenza della interpretazione delle Idee platoniche di Gadamer con quella di Friedländer. Quest'ultimo nega - come abbiamo anticipato e come meglio vedremo più avanti - che in Platone ci sia una vera e propria "teoria delle Idee". Gadamer ribadisce pro- 
prio tale concezione, in modo marcato. Dopo aver messo in rilievo come sia essenziale in Platone il «rivolgersi all'Idea, che sta oltre le brutte figure del sapere apparente», precisa: «Ma questo non significa neanche che Platone alla fine abbia una dottrina che possiamo apprendere da lui: la "dottrina delle Idee". E quand'egli critica questa dottrina nel suo dialogo Parmenide, ciò non vuol dire che egli allora abbia smarrito la strada. Ciò vuol dire piuttosto che le ipotesi delle "Idee" non era tanto una "dottrina", ma indicava piuttosto una direzione di ricerca, sviluppare e discutere le cui implicazioni è il compito della filosofia, cioè della dialettica platonica» (Verità e metodo 2, p. 487).

Inoltre, proprio come Friedländer, Gadamer nega che Platone ritenga il Bene conoscibile ed esprimibile, in quanto «il pensare rimanda sempre oltre se stesso» (ibidem).

Infine, ricordiamo che Friedländer sostiene la necessità di leggere e intendere Platone non come lo intendono «i Platonici». E Gadamer precisa: «Platone non era un Platonico» (Verità e metodo 2, p. 493).

Su alcuni particolari avremo modo di tornare anche più avanti. Ma quanto abbiamo detto dimostra ad abundantiam la tesi che sopra abbiamo sostenuto, ossia che questa opera di Friedländer non è solo un classico della letteratura secondaria per l'interpretazione di Platone, ma che si impone anche come un documento essenziale per la comprensione dell'ermeneutica di Gadamer, ossia di uno dei capitoli più importanti della storia della filosofia del secolo XX.

\section{Socrate come "destino" di Platone}

L'asse portante principale dell'interpretazione che Friedländer propone - come già sopra abbiamo accennato - sta nella identificazione della figura di Socrate con il fulcro del pensiero e dell'opera di Platone.

Socrate - egli dice - vive e parla in Platone. Proprio in Socrate Platone con gli occhi dell'anima «vede le Idee», e di conseguenza le intende e le esprime. 
Leggiamo alcune affermazioni di Friedländer particolarmente significative: «Platone si incontra con Socrate. Egli trova che il proprio volere, ancora del tutto indeterminato, "di dedicarsi subito alla vita pubblica", è guidato da quella ricerca in una particolare direzione. "Per me non c'è nulla di più urgente che divenire, quanto è possibile, virtuoso. $\mathrm{E}$ in ciò, credo, nessuno può aiutarmi più validamente di te". Così Alcibiade parla a Socrate nel Simposio, e così ha detto o sentito Platone di fronte a lui. E da Socrate egli ha inteso o creduto di dover intendere le parole che egli fa rivolgere da Socrate stesso al giovane Alcibiade in un altro dialogo: "tutti i tuoi piani non possono trovare il loro compimento senza di me; tanto io posso sulle tue faccende e su di te". Così vita e morte del maestro lo afferrano come suo proprio destino». (infra, p. 25)

Friedländer precisa inoltre: «In Platone quello che in Socrate era domanda si è fatto domanda e risposta, quel che in Socrate era vita si è fatto vita e dottrina. Socrate chiede: che cosa è il giusto? Egli fa che gli altri vedano che non lo sanno. Cerca la risposta nel concetto, ma la dà infine soltanto con il suo vivere e il suo morire. Platone vede e dà forma a questa vita e a questa morte. Ma vede di più. Trova anche la risposta come filosofema, vede, attraverso la figura di Socrate, l'idea. "Il giusto" come eterna essenza, come modello originale, veduto e mostrato: questa è la risposta alla domanda di Socrate, letta nella stessa realtà che si chiamava Socrate». (infra, p. 78)

Come si spiega, allora, il fatto che nei dialoghi dialettici Socrate cessa di essere protagonista, e che nelle Leggi risulta essere addirittura assente?

La risposta data da Friedländer è fra le più acute e ingegnose; e dal punto di vista interpretativo risulta essere assai più convincente di altre date da vari interpreti, e in particolare di quelle che parlano di «superamento di Socrate»o addirittura di «allontanamento da Socrate».

I ruoli svolti da Socrate nei dialoghi platonici sono i seguenti tre: 
1) in primo luogo risulta predominante il ruolo di protagonista in senso assoluto;

2) in secondo luogo, c'è il ruolo di colui che presenzia al dialogo, ma non entra in modo diretto nella questione discussa;

3) infine c'è anche un misterioso ruolo di "assente".

In realtà, questa triplice funzione risulta essere differente solamente all'interno dell'ottica drammaturgica, non però nella sostanza.

1) Quando Socrate è presente e svolge il ruolo di protagonista, domina la problematica e la sua finalità in tutti i sensi.

2) Quando è presente ma non entra direttamente nella discussione, significa che la questione che viene trattata per quanto possa essere importante - non affronta direttamente i problemi essenziali e ultimativi della vita; ma la presenza di Socrate risulta essenziale, in quanto indica che quei problemi che vengono discussi hanno un senso ultimativo solamente in riferimento ai problemi di fondo indicati da lui, e ribaditi appunto con la sua presenza.

Friedländer scrive: «Così appunto nei dialoghi che apparentemente mettono Socrate in disparte o lo fanno sparire completamente, diventa più chiaro quali idee egli rappresenti in Platone e quali no. Sarebbe evidentemente errato dire che, invecchiando Platone, la figura del maestro appaia, grado a grado, sbiadita ai suoi occhi. A ciò contraddirebbe il fatto che nel Filebo Socrate ha un significato importantissimo, per non dire del Fedro che lo porta sulla scena nella sua più vivace freschezza. E che egli nel Sofista e nel Politico e nella seconda parte del Parmenide non sia presente soltanto per abitudine, dovendosi limitare a un indifferente ruolo di comparsa, appare chiaro se lo si immagina assente: subito tutto cambia significato, perde il suo sottinteso, se non è più trattato in sua presenza».

Insomma: la via che si deve battere per giungere al fine che dà senso alla ricerca è quella che indica Socrate che non parla, ma è lì accanto che ascolta. 
3) L'assenza di Socrate nelle Leggi, infine, viene spiegata nel modo che segue: «Ma guardiamo ora anche l'altro lato! Nelle Leggi l'ospite ateniese riconosce esplicitamente che lo stato in cui domina la massima della comunione dei beni, donne e figli, è al primo posto, anche se sia adatto soltanto per dèi e figli di dèi, mentre la costituzione alla quale hanno messo mano solo si avvicina molto all'immortalità e tiene il secondo posto, questo certamente, essa sola ( $739 \mathrm{C}$ e ss.). Quindi non è che lo stato prima costruito sia messo da parte; esso è anzi da "tenersi incessantemente presente come modello". E così si può ben credere a ragione che Socrate non sia spinto via dal centro della visuale platonica, anzi domini là con immutata potenza e soltanto che lo stato delle Leggi sia con tutto il lavoro dedicatogli troppo allontanato da quel centro per presentarsi ancora sotto quel grande nome».

Si può quindi dire che "Socrate che parla", "Socrate che ascolta", "Socrate che è assente" simboleggiano «i gradi del pensiero di Platone» (infra, p. 162), vale a dire 1) il grado in cui Platone discute le questioni di fondo, 2) quello in cui tratta i problemi che non hanno un valore in sé e per sé, ma sono subordinati a un valore più alto, 3) e infine nelle Leggi quello in cui Platone per ragioni contingenti si allontana dalla "Città ideale" per trattare della "Città seconda", dalla quale Socrate rimane fuori di conseguenza, e alla "Città ideale" si fa solo un richiamo allusivo.

\section{II "dialogo" come "necessità" per Platone}

Come abbiamo già sopra rilevato, secondo Friedländer il vero creatore del dialogo socratico quale opera d'arte anche se altri si erano dedicati a esso in precedenza - è proprio Platone.

Senofonte, per esempio, presenta nelle sue opere socratiche numerosi dialoghi di Socrate, ma in essi sono quasi totalmente assenti quella energia vitale e quella forza purificatrice, nonché quel suggello imposto dall'aporia con tutte le sue implicazioni e conseguenze. 
Il "dialogo" fu per Platone una "necessità", in quanto ricreava la struttura stessa del pensare e del vivere di Socrate, sia pure portandola su un piano più elevato.

Friedländer precisa: «Ma c'è ancora un ultimo punto di vista dal quale la forma dialogica appare come la forma necessaria per Platone, perché, a partire da quella, la struttura della visione del mondo platonico, benché su di un piano più alto, sembra ripetere la struttura del mondo socratico. Per Socrate la risposta alla sua domanda si dissolve nel non-sapere. Per Platone la via dialettica conduce su, a ciò che è "al di là dell'essere". L'epekeina non è conoscibile e quindi neppure comunicabile: qui la via soltanto può essere preparata. Perciò il dialogo è una guida, passo per passo a un fine che, dietro il socratico non sapere e dietro l'inesprimibile della più alta visione platonica, è garantito come realtà dalla vivente persona del maestro. E come è proprio della fisionomia del dialogo socratico finire con il non sapere, così è proprio del dialogo platonico fermarsi dinanzi al punto estremo senza farlo vedere se non da lontano. Questo appare chiaro perfino nella struttura della Repubblica, e tanto più in ogni altro luogo, a ogni attento osservatore» (infra, pp. 190 sg.).

Il dialogo di Socrate è "natura", ossia una "forza di natura", "vita naturale". Il dialogo platonico diventa "opera d'arte", che non è semplice "immagine" di quel "modello", e quindi inferiore al modello, ma è come una icona che completa il modello e lo perfeziona a livello artistico.

Nel dialogo di Socrate ci possono essere elementi casuali e contingenti; nel dialogo platonico, al contrario, tutto diventa "necessario", e l'elemento apparentemente casuale diventa coefficiente essenziale e necessario in funzione dell'intero.

Di conseguenza, luogo, situazione e personaggi diventano elementi essenziali per il dialogo platonico e per la sua comprensione: «Così per la comprensione dei dialoghi dovrà essere esaminato, più di quanto perlopiù non avvenga, il luogo immaginato con gli atti materiali ivi raffigurati, per giungere al loro significato spirituale. Non come se si 
trattasse di allegorie nel senso dei neo-platonici - fra i quali tuttavia Proclo dirà cose molto assennate sullo scopo non semplicemente artistico né solo storico, bensì schiettamente filosofico del prologo nei dialoghi platonici. Quello che oggi l'interprete filosofo di Platone lascia al letterato e allo storico, deve essere compreso nel suo contenuto esistenziale. Perché quelle parti (prologo e discussione) non si trovano l'una di fronte all'altra per caso, già per il fatto stesso che gli scritti di Platone non appartengono a un ambito di arte naturalistica, ma di arte classica» (infra, p. 189).

La filosofia in Platone a livello di "arte" - così come in Socrate a livello di "natura" - non è solo il contenuto, ossia l'oggetto trattato dal dialogo, ma coincide con il dialogo stesso. Pertanto, "forma letteraria" del dialogo e "contenuto filosofico" in Platone coincidono strutturalmente, e quindi non sono distinguibili.

Tale coincidenza fra forma letteraria e contenuto del dialogo corrisponde alla coincidenza fra "realtà della vita" e "verità dell'essere", ossia fra "Idea" ed "esistenza".

Particolarmente significativa è la netta opposizione di Friedländer alla presa di posizione di Hermann Bonitz (che non pochi anche oggi continuano a condividere, almeno implicitamente), secondo cui nella lettura del Fedone (e quindi anche degli altri dialoghi) si deve lasciare da parte tutto ciò che è proprio della rappresentazione artistica del dialogo e puntare solo sul contenuto dottrinale del dialogo stesso. In realtà - rileva Friedländer - «la filosofia in Platone non comincia soltanto dove è fissato il primo punto della discussione dialettica, ma subito, quando ancora crediamo di essere fra chiacchiere di nessun impegno e ancora quando ancora crediamo di essere nella plasticità del gioco o del serio che fa da cornice» (infra, p. 266).

Dunque, i "dialoghi" di Platone coincidono con la "realtà del vivere", mentre cercano la "verità dell'essere".

Friedländer rileva inoltre - a giusta ragione - come, nella trasfigurazione poetica del dialogo naturale di Socrate, in Platone emerga anche una forte presenza esistenziale degli avversari, e quindi come la lotta ingaggiata 
contro di essi esprima anche una lotta contro le contrastanti forze presenti in Platone stesso. Fra i molti esempi che lo studioso adduce, uno è particolarmente significativo: «Se non ci fosse stato in lui qualcosa di Callicle, l'esprit fort, difficilmente l'avrebbe potuto presentare con tanta imponen$\mathrm{za}$, tale cioè che ci saranno sempre persone, specialmente giovani, incantate più dall'avversario sconfitto da Socrate che da Socrate stesso» (infra, p. 195). Questo significa che «Platone aveva da vincere, più di quel che taluno pensi, una natura dotata in modo straordinariamente ricco. Ma portava in sé anche Socrate, e delle lotte e delle vittorie che egli ci mostra, quelle veramente decisive sono accadute in lui stesso» (infra, p. 196).

Le conclusioni che Friedländer trae sono le seguenti: «Platone trovò ciò che Socrate "soltanto" cercava e insegnava a cercare. Ma si sa cosa vale la ricerca, se è ben condotta. "Nella domanda c'è la risposta, il sentimento che su di un certo punto si debba pensare qualcosa, qualcosa si debba correggere" dice Goethe. Dedicatosi alla dialettica socratica, a Platone si aprì la vista sulle forme eterne. Attraverso Socrate, e in Socrate, egli mirò il giusto in sé. Così sul nuovo gradino conquistato e appunto su quello soltanto poteva essergli dato questo compito: doveva accogliere la dialogica socratica, ma condurla oltre se stessa non a una fine scetticamente negativa, ma a rispondere alle domande poste e, se era possibile, alla conoscenza stessa dell'ente» (infra, p. 197).

Le tangenze di tali affermazioni con l'ermeneutica di Gadamer sono veramente consistenti.

\section{La grandiosa presentazione dei singoli dialoghi}

L'analisi e l'interpretazione dei singoli dialoghi sono veramente imponenti e di grande utilità, anche per chi non condivide il paradigma ermeneutico di Friedländer.

L'autore non procede secondo un tracciato dei dialoghi disposti l'uno dopo l'altro secondo un criterio puramente 
cronologico, ma li raccoglie in gruppi tematici, pur rispettando - nei limiti del possibile - la presunta successione cronologica.

Inizia con la trattazione di dialoghi giovanili, suddivisi nel modo che segue. Parte da un gruppo di dialoghi aporetici incentrati sulle tematiche di "areté", "philía", "kalón": Protagora, Lachete, Trasimaco (primo libro della Repubblica), Carmide, Eutifrone, Liside, Ippia maggiore. Prosegue con l'analisi di brevi dialoghi giovanili sul filosofo, sul sofista e sul poeta, che sono Ipparco, Ione, Ippia minore e Teagete. Passa quindi al gruppo dei dialoghi contenenti «autoritratto e maschera del filosofo»: Apologia di Socrate, Critone, Eutidemo, Cratilo, Menesseno, per concludere con Alcibiade maggiore, Gorgia e Menone, che vengono raccolti sotto il titolo «Il logos resta fermo».

Segue la trattazione dei grandi dialoghi considerati come una unità ben articolata. Dopo i grandi dialoghi intermedi Simposio, Fedone, Repubblica -, vengono trattati i dialoghi dialettici (Teeteto, Parmenide, Fedro, Sofista, Politico e File$b o-$, e a conclusione - sotto il titolo «Mitologia e Nomoteoria» - vengono trattati Timeo, Crizia e Leggi.

Il compito che lo studioso cerca di assolvere - come già diceva nel primo volume della sua opera sull'Alcibiade maggiore - è quello di tentare quelle di «concepire l'opera di Platone come un sistema stellare, su cui nessuna luce e nessuna forza può essere trascurata» (infra, p. 190).

Un particolare colpisce il lettore: Friedländer rivaluta non pochi dialoghi che molti studiosi respingono come poco significativi, e che considerano spuri.

Lo studioso afferma di seguire a questo proposito il principio metodologico di August Boeckh, secondo cui «soltanto la inautenticità e non mai la autenticità può essere dimostrata in maniera convincente - beninteso se mancano ragioni esteriori» (p. 270). Di conseguenza, la rivalutazione di dialoghi considerati spuri dai più è sempre accompagnata da affermazioni di carattere ipotetico.

$\mathrm{Ma}$, in realtà, la vera convinzione di Friedländer era invece che la cosa più difficile da dimostrare è proprio la non autenticità, salvo la presenza di precise ragioni esteriori. 
Gadamer ci riferisce quanto segue in Maestri e compagni (p. 35): «Friedländer preferiva impartire il suo insegnamento attraverso il seminario, come pure era sua consuetudine sostenere con acribia l'autenticità di opere o versi sospetti. Della sua fatica si sentiva ripagato abbondantemente se ogni tanto qualcuno si dichiarava convinto delle sue argomentazioni. Da Friedländer si imparava soprattutto quanto sia difficile dimostrare l'inautenticità di un'opera e come abbia ragione Hegel quando dice: "Gli argomenti sono a buon mercato come le more". Non è certo che le argomentazioni di Friedländer rendessero più autentici i testi, attestano però, pur nella loro esagerazione, come da lui si imparasse in maniera incomparabile a disciplinare il proprio senso linguistico, quell'orecchio interno cioè, senza il quale non è possibile, in generale, alcun giudizio letterario».

Si può ben dire che - proprio in qualità di filologo di alta classe - Friedländer capovolge radicalmente quella tenden$z a$ assai diffusa nei filologi tedeschi dell'Ottocento di contestare l'autenticità di tutta una serie di dialoghi, tanto che, a conti fatti, sono stati ritenuti autentici solo pochissimi. Zeller elencava come dialoghi non contestati solamente i seguenti sei: Protagora, Fedro, Simposio, Gorgia, Teeteto e Repubblica. Ma dopo Zeller si è tentato di atetizzare anche il Protagora.

Dunque, si è dubitato perfino dell'autenticità di un dialogo come il Fedone. Inoltre, anche filologi di grande famacome Ueberweg, Schaarschmidt e Windelband - hanno negato l'autenticità del Parmenide, ossia del dialogo più studiato dai Neoplatonici.

Le analisi più interessanti e motivate di Friedländer sono proprio quelle dedicate ai dialoghi minori e considerati inautentici, che, di conseguenza, dai più vengono considerati e studiati in una ottica in partenza scorretta.

Richiamiamo qui - per ragioni di spazio - solo alcuni esempi, a nostro avviso particolarmente significativi.

Sull'Alcibiade maggiore Friedländer si era già impegnato a fondo nei volumi del 1921 e 1923, di cui abbiamo già detto sopra. A giusta ragione lo studioso rileva - tra l'altro 
- che nel dialogo ci sono pagine che solo un Platone poteva scrivere.

Anche sull'autenticità dell'Ipparco Friedländer ha pochi dubbi. Dopo attente e belle analisi egli trae le seguenti conclusioni: «Non ci sono ragioni per assumere in generale come possibilità che in questo dialogo sia all'opera un imitatore tardo finché non si sia in grado di trovare nel linguaggio, nella terminologia e nelle problematiche filosofiche, o nella forma artistica, il più piccolo indizio di un'epoca successiva di composizione. Intorno al 400 a. C. il dialogo risulta un prodotto naturale di un'epoca; intorno al 330 un anacronismo incomprensibile. Se l'opera è una di quelle scritte all'inizio così come la semplicità della struttura e il fatto che i motivi concettuali non siano ancora sviluppati indicano, è difficile pensare a un autore che non sia Platone, con le cui opere troviamo connessioni dappertutto nel dialogo. Non è forse, allora, l'ipotesi più probabile che l'I $p$ parco sia uno dei primissimi dialoghi di Platone?» (infra, pp. 530-531).

Di grande finezza è l'interpretazione del Teagete, dai più considerato spurio, e su cui Friedländer trae invece le seguenti conclusioni: «In nessun luogo platonico si trova espressa più chiaramente l'educazione attraverso l'amore e la presenza immediata. Neppure potrà esserci (dove ci sia anche Socrate) un muto star seduti l'uno accanto all'altro. Il dialogo è una parte indispensabile della presenza socratica. In ogni caso non possiamo obiettare a quanti vedono la presenza di forze magiche e quindi considerano questi temi non platonici. Noi non vediamo qui nulla di magico, o quantomeno nulla che abbia a che vedere con quel tipo di magia che Faust voleva "rimuovere dal suo cammino". C'è solo la magia del grand'uomo e dell'educatore. Inoltre riteniamo che questioni del genere non possano esser decise solo con lo strumento della critica filosofica ma richiedano una certa ampiezza di vedute» (infra, p. 560).

Dell'Ippia minore - che dagli studiosi non viene respinto come spurio solo perché Aristotele lo cita espressamente, ma che, come dice il nostro studioso, ha tutti quei caratteri 
per cui altri dialoghi analoghi sono respinti - viene fornita una delle più toccanti e penetranti esegesi: «In ogni caso Socrate, che qui vediamo "ingannare" il suo oppositore apparentemente alla maniera dei sofisti, è la testimonianza vivente del fatto che chi sa la verità può ingannare meglio di chi la ignora, e chi inganna come fa Socrate, "volontariamente", è migliore di chi lo fa involontariamente, e non solo nel senso di sfoggiare una maggiore abilità ma anche nel senso di essere più vicino all'agathon. Così il dialogo in oggetto è il più puro ritratto del modo di esistenza socratico, tanto spesso descritto (insieme alle questioni più disparate) nei dialoghi giovanili come nelle opere più mature di Platone: il ritratto del maestro sovrano di tutte le tecniche sofistiche con lo sguardo rivolto al "Bene" o dell'ambiguo educatore con la mente rivolta allo scopo da raggiungere» (infra, p. 551).

Per quanto concerne il Menesseno Friedländer offre la migliore interpretazione che finora abbiamo incontrato. La tesi interpretativa di fondo a nostro avviso è esatta: nel dialogo Platone dimostra di saper essere un vero e competente retore, e anticipa in piccolo quello che in grande, per quanto concerne la retorica, dimostrerà nel Fedro.

Per quanto riguarda le analisi e le interpretazioni dei dialoghi minori e della giovinezza in Friedländer si trova di più di quanto si trova in altri interpreti in generale, con osservazioni e motivazioni spesso convincenti; invece, per quanto riguarda le analisi e le interpretazioni dei dialoghi maggiori si rimane spesso a bocca asciutta, e ci si aspetterebbe di più.

Questo avviene a motivo della negazione dell'esistenza di una platonica "teoria delle Idee", e per una sorta di "riduzionismo teoretico" del pensiero di Platone tipico del paradigma ermeneutico di Friedländer.

Va comunque detto che le analisi dei singoli dialoghi è sempre fatta con grande accuratezza; ma è spesso debole l'interpretazione dei passi vari metafisici e speculativi.

Per rendersi conto di risultati ottenuti con differenti approcci derivanti da un differente paradigma ermeneutico si vedano per esempio: Thomas A. Szlezák, Platone e la scrit- 
tura della filosofia, Milano 1988; $1992^{3}$ e Michael Erler, Il senso delle aporie nei dialoghi di Platone, Milano 1991.

$\mathrm{Va}$ comunque detto che dalle analisi di Friedländer dei singoli dialoghi si ricava in buona misura il meglio che sia stato prodotto nell' ambito del paradigma interpretativo tradizionale di Platone.

Inoltre, bisogna altresì rilevare che lo studioso ha fatto ciò che nessun altro è riuscito a fare con la stessa intensità: ha evidenziato numerosissime tangenze dei vari dialoghi fra di loro, ha indicato tutta una serie di richiami incrociati, mostrando davvero - per usare la sua stessa metafora come l'insieme degli scritti platonici sia come un sistema stellare, e ha cercato di riprodurre in modo magistrale le caleidoscopiche figurazioni che esso assume e le sue luci e controluci che si incrociano.

\section{L'ironia" in Socrate e in Platone}

Molto fini sono le pagine dedicate alla "ironia"; e giusta è l'affermazione di Friedländer secondo cui il problema da molti studiosi non è stato adeguatamente considerato, e solo pochi hanno mostrato di prendere coscienza del senso metafisico dell'ironia stessa.

Friedländer ammette che l'ironia in Socrate e in Platone ha un valore "metodologico", ma egli prende soprattutto in considerazione il valore "esistenziale" di essa, e scrive: «Hegel ha considerato l'ironia socratica come un lato del "metodo socratico" (l'altro è la maieutica): "Quel che Socrate voleva ottenere era che gli altri si spiegassero, che esponessero i loro principi". Indubbiamente viene così individuato qualcosa di essenziale della sua opera. $\mathrm{Ma}$ si fraintenderà sempre il fenomeno - e nella letteratura filosofica della quale Hegel sembra avere determinato il concetto, questo fraintendimento non è raro - se si ritiene regola pedagogica intenzionale ciò che poteva educare veramente soltanto in quanto era necessariamente così. La pura ironia contiene in sé questo contrasto: se da un lato vela illudendo, dall'altro 
senza riserva esprime l'ente. Come non dipendeva da Socrate l'insieme del suo aspetto esterno di Sileno e l'interna bellezza, ugualmente non stava in lui di nascondere con un atto di arbitrio il suo sapere dietro un non sapere: l'uno e l'altro sono legati in un eterno moto circolare o in un'oscillazione di pendolo» (infra, pp. $170 \mathrm{sg}$.).

In effetti, nell' "esistenza ironica" di Socrate il "sapere" nel suo essere "esistenzialmente incarnato" balza al di sopra dell' "ignoranza": il "non-saputo" nel logos è superato dal "saputo" nella vita.

Pertanto, «a questo punto l'ignoranza si converte in un sapere che è l'estremo sapere; il non sapere nel logos era fondato infatti su di una esperienza vitale del non saputo. E dove può trovarsi un sapere più profondo di quando si è esistenzialmente, nel vivere e nel morire, proprio quello che nella parola non si è mai cessato di cercare?» (infra, p. 171).

Che differenza c'è, allora, fra l'ironia incarnata da Socrate nella sua esistenza, e quella platonica?

Ancora una volta si può dire che, mentre l'ironia socratica è "natura", quella di Platone è espressione ed evidenziazione articolata in dimensione artistica e metafisica di quella "natura". Lo studioso precisa: «Il Socrate platonico porta in sé il mistero socratico e l'ironia socratica che esprime ed esaspera quella antitesi fra il non sapere a parole e il sapere nella vita vissuta, ma porta anche in sé, andando innanzi negli anni con Platone e in Platone, il mistero platonico e l'ironia platonica» (infra, p. 174).

Platone simboleggia spesso proprio mediante la stessa ironia ciò che ha di più elevato da dire.

Dunque, l'ironia "vela" e nello stesso tempo "disvela" il mistero platonico. Come in una statua della grande arte greca la veste vela e nello stesso tempo mostra ciò che è velato, così, analogamente, l'ironia platonica vela e disvela le forme, e anche ciò che sta al di sopra dell'essere, ossia il Bene.

In effetti, Platone in non pochi passi-chiave dei suoi dialoghi con la sua ironia si arresta davanti al punto estremo, ma nello stesso tempo in qualche modo lo indica e lo fa vedere da lontano. 
Pertanto, mediante l'ironia del "Socrate zetematico", Platone ci rappresenta ciò che nella sua esistenza si impone come "simbolo della realtà del bene" e ad un tempo della sua "ineffabilità".

Le belle pagine di questo capitolo andranno però completate con le fini analisi fatte da Jan Patocka nel suo Socrate (Bompiani 2003), che approfondisce alcuni aspetti della realtà esistenziale della vita ironica di Socrate, e inoltre anche con quelle di Gregory Vlastos, che approfondisce invece il significato metodologico di quella che egli chiama "ironia complessa" (Socrate il filosofo dell'ironia complessa, Firenze 1998).

\section{La teoria delle Idee}

La posizione "riduzionistica" assunta da Friedländer nei confronti della teoria platonica delle Idee, che si spinge oltre i giusti limiti, proprio a livello critico-interpretativo, è stato messo subito in rilievo da alcuni studiosi.

Adolfo Levi (che è stato uno dei maggiori studiosi italiani di Platone) in una recensione, pubblicata nella «Rivista di filologia e di istruzione classica» nel 1932, poco dopo l'uscita del secondo volume, scriveva: «ll punto più debole del volume mi appare il modo in cui esso parla (o, quasi, non parla) della teoria delle Idee. Già nel primo volume il Friedländer sosteneva che ciò che gli studiosi chiamano con questo nome è soprattutto una loro costruzione, di cui pochissimi elementi si trovano negli scritti platonici, che non offrono alcuna dottrina, alcun sistema; a suo parere, ciò risulta dalla Lettera VII, in cui Platone, riferendosi al mondo delle Idee, dichiara che non esiste e non esisterà mai alcuno scritto sulla propria dottrina, perché essa non è in alcun modo esprimibile. In questo volume, che rapidamente accenna alla stessa tesi (...) si può dire che delle Idee platoniche non si parla in modo preciso, sicché l'argomento rimane quasi completamente indeterminato. Senza ritornare alle osservazioni che ho fatto sulle affermazioni del Friedländer recensendo il suo primo volume [si veda il passo che riportiamo 
al paragrafo che segue], mi limito a notare che secondo Platone nessuna ricerca che miri seriamente alla scoperta del vero può esprimersi adeguatamente colle parole; rispetto alla teoria delle Idee le difficoltà sono maggiori che negli altri casi, in cui si considerano oggetti diversi, ma ciò non impedisce che il linguaggio scritto possa servire, sebbene in modo imperfetto, a tradurre l'apprensione intellettuale. Ciò del resto è provato dal fatto che Platone ha parlato del mondo ideale ben più largamente di quanto voglia riconoscere il Friedländer. Si astenne dall'esporre per mezzo di trattazioni scritte soltanto la sua ultima dottrina (la teoria delle Idee Numeri, non menzionata dal Friedländer, che doveva costituire una sistemazione organica della speculazione platonica)». Levi conclude con la giusta osservazione che, siccome Platone parlava di tali dottrine nei corsi di lezione all'interno dell'Accademia, doveva essere in ogni caso convinto che si potesse giungere alla conoscenza delle realtà supreme, sia pure dopo un lungo e faticoso processo di ricerca.

L'affermazione che Friedländer fa sulla genesi della dottrina delle Idee ha certamente qualcosa di vero: Platone ha veduto l'eidos nella vita di Socrate, e in generale cercando il vero Stato, quindi cercando il nuovo uomo per il nuovo Stato. Lo studioso scrive: «D'altronde non è un caso che Platone abbia parlato per primo, per quanto sappiamo, dell'occhio dell'anima, che egli lo faccia quando ha dinanzi a sé gli scopi ultimi del suo filosofare; e non casualmente, anche dove non impiega la parola, il suo linguaggio metaforico è mitico e appartiene allo stesso ambito. Egli dice chi gli abbia insegnato la via, ponendo nei suoi dialoghi Socrate come unica guida verso questo scopo. Anche nel mito della caverna, nella Repubblica, l'uomo che è riuscito a liberarsi dalle catene porta i tratti di Socrate. Infatti quando ritorna dai prigionieri e vuole "scioglierli e condurli fuori", ecco che lo ucciderebbero, solo che potessero averlo fra le mani. Platone non poteva dire più chiaramente chi lo ha convertito e condotto lassù dove, prima, ha imparato a conoscere vere ombre di cose vere, poi le loro 
immagini riflesse, poi quelle cose "in sé" e il "sole". Dunque per mezzo di Socrate e, per così dire, in lui, ha mirato con l'occhio dell'anima il "giusto in sé" o piuttosto ancora "il giusto" e anche "il coraggio", "la temperanza", "la saggezza", in generale "le virtù" e "la virtù". E vero che tutti già parlavano di essa, se fosse o no insegnabile e ciascuno intendeva qualcosa di diverso con quel chiuso nome, più volentieri quel che gli aggradava. Ma Socrate è l'unico che non solo la ricerca con parole - per quanto si eserciti in tale ricerca molto più seriamente e continuamente degli altri - ma che con la sua vita e con la sua morte garantisce che essa sussiste; e nell'essere socratico "l'occhio dell'anima" di Platone vede quelle immagini, quelle forme, quelle figure» (infra, pp. 28 sg.).

Dunque, Platone ba veduto e trovato con l'"occhio dell'anima" ciò che Socrate aveva vissuto e insegnato a cercare. Si tratta, pertanto, di una intuizione e di una esperienza originaria di vita. Le conseguenze che lo studioso trae sono di grande importanza, e dimostrano anche la fallacia dell'interpretazione fornita da Aristotele nel primo libro della sua Metafisica, secondo cui l'Idea platonica deriverebbe dalla scoperta socratica del "concetto", e non sarebbe se non l'ipostatizzazione ontologica del concetto stesso: «Lidea ha una storia di più di due millenni e nessuna parola del linguaggio filosofico è stata tormentata di più dal pensiero dei secoli. Solo l'eidos platonico non è filosofia nata dalla filosofia, come da Platone in poi, e essenzialmente per causa sua, ogni studio sulle idee. Appunto per questo è necessario rimetterne in luce il concetto, per così dire, nella sua innocenza» (infra, p. 32).

Friedländer precisa: «Indubitabile è questo fatto di somma importanza e quasi sempre disconosciuto: che la sua filosofia non gli è data dai precedenti sistemi. Non appena gli occhi gli si aprirono all'eidos, tutte le forze del suo essere con la loro prodigiosa tensione furono applicate in quest'unico senso e per la prima volta ora Platone divenne, in un significato affatto nuovo, filosofo. Ed è assai più da cercare la legge secondo la quale intorno a quel punto di unità si cri- 
stallizzò la materia trattata, che non l'ordine storico in cui tutto questo accadde» (infra, p. 35).

Platone non ha, comunque, tenuto per sé ciò che aveva veduto e sperimentato in Socrate, e ha voluto rivelarlo agli altri: ha voluto aprire anche agli altri gli occhi, perché essi pure lo potessero vedere.

Ma se si ammette questo, come si può negare - come fa il nostro studioso - che Platone avesse una sua "teoria delle Idee"? Per comunicare agli altri ciò che aveva visto, doveva in ogni caso esprimere la sua visione mediante il logos, e quindi esprimere una teoria.

In effetti, Friedländer stesso va contro se stesso, nel momento in cui mostra come, per esprimere mediante il logos la propria intuizione, Platone si è rifatto alla dottrina di Parmenide sull'essere: «L'intera costruzione del suo mondo dell'essere e delle forme di conoscenza corrispondente ai gradi è rigorosamente parmenidea» (infra, p. 38).

Inoltre, egli chiama anche in causa Eraclito e la sua teoria dei nessi strutturali fra l'Uno e i molti e l'armonia dei contrari: «Ma c'era tuttavia una più alta veduta, nella quale questa dualità si riduceva all'unità. "Uno è il tutto", "Concorde e discorde, consonante e dissonante, da tutto uno e da uno tutto": così Eraclito. E Platone: l'idea, che è una e le cose singole che sono molte, si richiamano reciprocamente. L'idea fornisce alle cose partecipazione all'essere, esse a loro volta tendono alla perfezione dell'idea. Solo se l'una non è senza le altre "il tutto è collegato con se stesso". Non era questo eraclitismo più puro persino di quella annacquata e sofistica, abusata teoria del flusso di tutte le cose?» (infra, p. 40).

Infine, lo studioso chiama in causa Pitagora, da cui Platone trasse il concetto di cosmos, ossia la concezione della ordinata compagine non solo dell' "Universo", ma anche della "Polis" e dell'“anima", e quindi una organica visione dell'uomo nel tutto inteso come un micro-cosmo in un macro-cosmo.

Emerge, di conseguenza, un innegabile tracciato categoriale nascente, e quindi una griglia di concetti secondo cui 
quell'originaria intuizione del mondo delle Idee viene espressa.

Del resto, le numerose pagine che dal Fedone in poi parlano delle Idee non solo per velate allusioni, smentiscono drasticamente l'interpretazione "riduzionistica" di Friedländer. Il lettore interessato potrà vedere la descrizione di tale griglia concettuale che si ricava dai dialoghi platonici nella nostra opera Per una nuova interpretazione di Platone (Vita e Pensiero, Milano 200321) con la relativa dettagliata documentazione (cfr. parti terza e quarta, passim).

\section{La dottrina dell'anima e della sua immortalità}

Come per la teoria delle Idee, Friedländer assume una posizione "riduttivistica" anche nei confronti della teoria platonica dell'anima.

Egli ritiene che Platone sull'anima sia stato influenzato soprattutto dagli Orfici, al punto da affermare che egli possa in qualche modo considerarsi un «teologo orfico», e scrive: «Sulla sua essenza e sul suo destino parlavano il trasparente mito dei pitagorici e dei seguaci di Orfeo. La loro dottrina sull'anima, Platone l'ha accompagnata con la sua più forte simpatia e ce ne è tanta nei suoi scritti da render concepibile l'idea che egli sia stato, fra tutt'altre qualità, anche un «teologo orfico». In altri termini la dottrina dell'immortalità $e$ dell'eternità dell'anima individuale, difficilmente potrebbe armonizzarsi con la dottrina delle idee; cioè Platone in effetti avrebbe preso a prestito quell'articolo di fede dai teologi che glielo offrivano già pronto. $\mathrm{Ma}$ anche se qui fosse rilevata una contraddizione nel sistema, non è affatto buon metodo spiegarla unificando dottrine originariamente separate. Eppoi in Platone! Certamente egli stava sempre in ascolto cercando di cogliere affinità di toni, ma era il più restio ad adattare bene o male al suo sistema dottrine estranee. Troppo poco ci si è resi conto che Platone in generale nulla "insegna" direttamente dei destini dell'anima. Socrate ne discute all'interno di miti che sono parte integrante della 
drammaturgia platonica. E se si richiama ai sacerdoti dei misteri e ai teologi, vien cosi designata la derivazione di quelle rappresentazioni mitiche, ma in nessun modo è detto che cosa erano in sostanza per lui. Se qui è lecito avanzare un'ipotesi, si dirà che avevano una profonda realtà simbolica o eran per lui forme, immagini, espressioni di quello che preferiva dire nel proprio linguaggio. Ma si sbaglia se si fa di tutto ciò la base per una fisica o una storia platonica dell'anima» (infra, p. 43).

$\grave{E}$ appena il caso di ricordare come i dati di fatto smentiscano la tesi di Friedländer, secondo cui la dottrina dell' anima e dell'immortalità «difficilmente potrebbe armonizzarsi con la dottrina delle Idee». Infatti, dal Menone in poi i testi dicono il contrario.

Si può certamente affermare che Platone vide nella vittoria di Socrate sulla morte una garanzia dell'immortalità dell'anima, così come in lui vide l'eidos. Ma si deve dire, nello stesso tempo, anche che tutte le prove che Platone fornisce sull'immortalità si fondano proprio sulle Idee. Pertanto, la dottrina dell'anima in Platone non solo si armonizza con la dottrina delle Idee, ma è ontologicamente e strutturalmente inscindibile da essa.

D'altra parte, lo stesso Friedländer in modo curioso smentisce se medesimo: pur affermando che nessuna delle prove a favore dell'immortalità raggiunge l'obiettivo, deve ammettere quanto segue: «Ma le prove dell'immortalità delle quali nessuna con buon fondamento giunge all'ultimo scopo, rendono evidente un'altra cosa. L'eternità dell'anima è garantita per Platone dall'essere dell'idea. Solo per l' "amico delle idee" ha un senso parlare dell'immortalità. Se l'anima umana secondo la propria natura è così fatta da conoscere l'eterno essere, essa allora deve - perché l'uguale è conosciuto dall'uguale - avere un essere a guisa delle forme eterne» (pp. 44 ss.).

Il capitolo dedicato all'eidos conclude addirittura con l'affermazione che l'anima diventa eterna in funzione dell'eidos che ha visto, e che anima e eidos stanno fra di loro in rapporto di necessità. 


\section{L“arrheton", il suo oggetto di riferimento e il suo significato}

Friedländer ha poi incentrato la sua attenzione sul concetto di "arrheton" - ossia sull" "indicibile" e "inesprimibile" - facendo una serie di rilievi di grande finezza, ma nello stesso tempo cadendo in errori interpretativi di rilievo: 1 ) per quanto riguarda l'oggetto cui "arrheton" si riferisce e 2) per quanto concerne la determinazione dell'area semantica che il termine ricopre.

1) Come abbiamo già sopra ricordato, l' "arrheton" secondo Friedländer si riferisce al mondo delle Idee in generale.

I motivi che egli adduce si connettono strettamente con la tesi che abbiamo sopra illustrato, secondo la quale non ci sarebbe in Platone una teoria delle Idee, vale a dire non ci sarebbe un sistema che presenti l'ordinamento delle Idee e ne chiarisca il rapporto con il mondo. Se si passa dalle trattazioni dei moderni sulla teoria delle Idee, nei dialoghi platonici si trova ben poco di quanto in quelli si legge. Scrive Friedländer: «Dopo aver studiato gli scritti dei moderni pensatori sulla "dottrina platonica delle idee", quando si ritorni ai dialoghi, per prima cosa ci si dovrà meravigliare considerando quanto poco si trova esposto a proposito di quel punto principale della filosofia platonica. Nei primi dialoghi, fino al Gorgia compreso, si posson cogliere solo accenni al fatto che c'è qualcosa come un bene in sé, un sommo amore, un bello veramente bello. Il Fedone accumula (almeno apparentemente) prove dell'immortalità che si basano su di un mondo delle idee, presupponendolo esistente. Nel Simposio si trova descritto il cammino che conduce su alle eterne essenze, nel Fedro un mito intessuto attraverso lo spazio sede delle idee, nella Repubblica si trova descritta l'ascesa attraverso le scienze e raffigurata con una grande metafora la via, l'essenza, gli effetti, nel Parmenide le aporie della dottrina, nelle altre opere della tarda età i suoi presupposti logici e le sue conseguenze sono discussi a fondo. Ma una "teoria" esposta in 
sé e per sé non si trova; nessun sistema che racchiuda in un ordine queste forme, che ne procuri la conoscenza scientifica, che chiarisca il loro rapporto col mondo del divenire» (infra, p. 78).

Si può ben concedere che, in realtà, in certe trattazioni sulla dottrina platonica delle Idee di autori moderni si trova veramente "troppo tanto", ma si deve anche dire che in Friedländer si trova davvero "troppo poco": lo studioso commette infatti un errore uguale e contrario a quello commesso dagli avversari. Nei dialoghi citati nel passo sopra letto si trova ben di più di quanto egli crede di vedere. In particolare nei dialoghi dialettici e soprattutto nel Filebo - che non viene citato nel passo - delle Idee viene presentata addirittura la struttura ontologica in generale e in particolare.

La posizione assunta dallo studioso si comprende bene dal punto di vista ermeneutico: il suo paradigma interpretativo urtava contro alcune precise pagine dei dialoghi citati, oltre che del non citato Filebo, le quali costituivano l'insuperabile ostacolo di un "controfatto". Di conseguenza, Friedländer ha cercato in vario modo di arte-fare quel controfatto fino al punto di dis-farlo, sia nella interpretazione generale, sia nelle analisi dei singoli dialoghi.

Però bisogna ammettere che ha svolto questa operazione con grande intelligenza e abilità.

In realtà, l'"arrheton" non riguarda il mondo delle Idee in quanto tale, ma solamente l'Idea del Bene.

Dopo aver affermato che in Platone non c'è una teoria delle Idee presentata in maniera precisa, lo studioso scrive: «Perché sia così, ce lo dice la Lettera VII in quell'importantissimo passo a cui ci riporta sempre il nostro esame. Non c'è nessuno scritto di Platone, non può, né potrà mai esserci alcun suo scritto su ciò che nella sua dottrina è veramente serio per lui "perché in nessun modo è esprimibile come gli altri oggetti del sapere". Nessun dubbio che si tratta della sfera delle idee» (infra, p. 78).

Invece, proprio la Lettera VII fa richiamo all'oggetto specifico delle dottrine non scritte, che non è la dottrina delle Idee ma la teoria dei Principi primi e supremi. 
I termini e le espressioni con cui Platone indica i contenuti cui fa riferimento in tale lettera sono inequivocabili, come per esempio i seguenti: l'«intero o il tutto» (

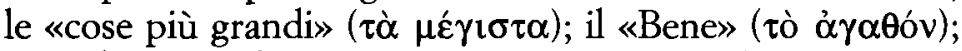
«ciò che riguarda i Principi primi e supremi» ( $\tau \dot{\alpha}$ ók $\rho \alpha \kappa \alpha i$ $\pi \rho \hat{\omega} \tau \alpha)$. Si tratta, dunque, di espressioni che riguardano ben più che le Idee in generale, proprio i Principi primi e supremi (si veda quanto precisiamo in Per una nuova interpretazione di Platone, pp. $101 \mathrm{sg}$.$) .$

In conclusione, "arrheton" non si riferisce al mondo delle Idee in generale, ma ai Principi primi e supremi.

Va altresi precisato che nella Lettera VII Platone non nega un suo scritto sulla dottrina delle Idee, ma appunto sui Principi primi e supremi.

2) Inoltre, anche il significato specifico di "arrheton" non viene correttamente inteso da Friedländer.

In effetti, Platone scrive: «La conoscenza di queste cose non è affatto comunicabile come le altre conoscenze, ma dopo molte discussioni fatte su queste cose, e dopo una comunanza di vita, improvvisamente, come luce che si accende da una scintilla che si sprigiona, essa nasce nell'anima e da se stessa si alimenta» (Lettera VII, C-D). Se viene letto con attenzione, il testo non dice che la conoscenza di tali cose (ossia dei Principi primi e supremi) sia affatto incomunicabile in sé e per sé, ma precisa che «non è affatto comunicabile come le altre conoscenze ( $\dot{\omega} \varsigma \alpha \alpha \lambda \lambda \alpha \mu \alpha \theta \dot{\eta} \mu \alpha-$ $\tau \alpha)_{\gg}$. E pertanto essa è di per sé comunicabile e scrivibile, anche se in maniera del tutto diversa, come viene puntualmente precisato.

Inoltre, non si può affatto affermare che il Bene non sia oggetto di conoscenza.

Infatti, in primo luogo Platone espressamente chiama

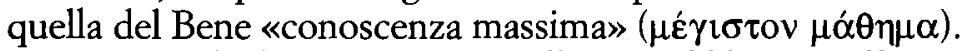

In secondo luogo, sempre nella Repubblica, si afferma senza mezzi termini che è dialettico solamente colui che sa rendere ragione dell'essenza di ciascuna cosa, e che questo vale anche per il Bene, e si precisa: «chi non è capace di definire l'Idea del Bene con il ragionamento, astraendola ('ُ $\dot{\varphi} \varepsilon$ - 
$\lambda \omega ́ v)$ da tutte le altre», resterà addormentato in questa vita $\mathrm{e}$ terminerà il suo sonno nell'Ade (VII, 534 B 3-D 1).

Si tenga presente anche il fatto che nel libro VI della $R e$ pubblica Platone non dice di non conoscere l'essenza del Bene, ma al contrario dice di avere convinzioni su di essa (cfr. 506 D 8-E 3; 509 C 3 sg.), e precisa di non volere comunicare il suo pensiero in tale sede. Afferma, inoltre, di volere pagare solamente gli "interessi" del debito. Di conseguenza, ciò che egli presenta è solamente il "frutto", ossia il "figlio"; però precisa di voler presentare gli interessi del debito in giusta proporzione.

Ben si può intendere come dagli interessi pagati in giusta proporzione sia possibile risalire al capitale, ossia risalire dal figlio al padre.

E Platone fornisce addirittura indicazioni di notevole portata che aiutano il lettore in questa risalita dal figlio al padre. Infatti, dopo la descrizione del figlio, ossia dell'immagine del Bene come Sole, Platone si spinge in modo cospicuo oltre l'immagine (pagando, oltre agli interessi, anche parte del debito): «E così anche ai conoscibili dirai che proviene dal Bene non solo l'essere conosciuti, ma che anche l'essere e l'essenza provengono loro da questo, pur non essendo il Bene essere, ma ancora al di sopra dell'essere, essendo superiore in dignità e potere» $(509 \mathrm{~B}-\mathrm{C})$. Al che Glaucone risponde: «Apollo! Che divina superiorità». $\mathrm{E}$ "Apollo", come sappiamo da Plutarco e da Plotino, è simbolo pitagorico dell" "Uno".

Pertanto il Bene viene indicato con allusione assai forte come «Uno» (tra l'altro, si tenga presente che solo in questo testo Apollo viene usato in forma esclamativa).

Ma c'è ancora di più.

Platone - mettendo in atto un gioco drammaturgico che consiste in quello che Friedländer chiama «dislocamento ironico del centro di gravità dell'opera (infra, p. 177)» (dislocamento ironico che lo studioso ha ben compreso per altre opere ma non per la Repubblica) -, nei libri IV e V indica espressamente l'essenza del Bene della Città - proprio ciò che il dialogo ricerca - appunto nell'Uno: indica il massimo 
Bene della Città in «ciò che la lega insieme e la fa una» e il massimo male in «ciò che la divide e invece di una ne fa molte» (cfr. IV, 422 E-423 B; V, 462 A-B).

In conclusione, il Bene è "arrheton" in senso ben diverso da ciò che è ineffabile e indicibile in senso assoluto: è conoscibile e dicibile in maniera molto diversa rispetto alle altre cose.

\section{Platone e la mistica}

Questa presentazione di Friedländer del pensiero platonico come tensione e come ascesa verso l'“arrheton", e l'improvviso accendersi di quella luce nell'anima che fa vedere il Bene, poteva portare a concludere che nel nostro filosofo è presente una forma di "misticismo".

Friedländer scrive: «Ciò che è sommo, "raramente si mostra, e a uomini rari". Si può bene indicare il cammino della scienza; è quanto avviene nella lettera. $\mathrm{Ma}$ altro è indicarlo, altro compierlo. E alla fine di esso c'è qualcosa di ineffabile. Potrebbe darsi che qui fosse accennato un ambito mistico e un personale mezzo di salvazione, purché sotto il misticismo non si immagini uno stato di ebrietà e di estasi e sotto la via di salvazione qualcosa di pretesco» (infra, pp. 79 ss.).

Ma subito appresso egli approfondisce il problema del misticismo, per giungere alle conclusioni che «Platone non è un mistico», che anzi addirittura si distacca nettamente dal misticismo, e scrive: «Così il mistico conosce un cammino soltanto: allontanarsi dall'bic et nunc, in cui al contrario l'uomo classico trova la sua compiutezza. E se Platone, da un punto di vista storico, col suo moto verso l'epekeina, avvia quella fuga ed è da cercare in lui l'origine di molta speculazione mistica, purtuttavia egli appartiene troppo al tipo classico, perché non debba esser veduto, in fondo, in contrapposto a ogni mistica. Si potrebbe infine ancora una volta chiarire questa opposizione confrontando le punte più alte della speculazione platonica e plotiniana, come abbiamo 
prima chiarito l'eros platonico commisurandolo a quello plotiniano» (infra, p. 100).

Va però rilevato che per "misticismo" Friedländer intende una esperienza spirituale del tutto "a-razionale" o "iporazionale".

Per la verità, il misticismo può assumere forme di questo genere, ma anche forme differenti in cui si manifesta come esperienza non irrazionale, ma piuttosto meta-razionale.

E la perfetta esperienza mistica che assume forma di conoscenza iper-razionale avviene proprio mediante la unificazione di soggetto conoscente e oggetto conosciuto.

Parlando di Plotino, Friedländer scrive: «In movimenti sempre nuovi è espressa l'unificazione, vien superata la contrapposizione: "Correndo là nell'intimo ha tutto, e lasciando addietro la percezione per timore di essere un altro, là è uno". "Bisogna abbandonarsi nell'intimo e in luogo di essere uno che vede, divenire spettacolo di un altro spettatore". Così dall'attività del contemplante è nata una situazione in cui egli si abbandona senza volontà alla emanazione e si fa prendere e emanare da essa. "Finché lo vede come un'altra cosa non è ancora nel bello; diventato quello, allora è massimamente nel bello". Dopo che si è veduta la energia di questa unione, ritorniamo ancora a Platone. Orbene in esso l'io e l'oggetto rimangono in rigorosa opposizione» (infra, pp. $101 \mathrm{sg}$.).

$E$ invece, nel finale del discorso di Diotima sull'Eros nel Simposio, si allude in modo chiaro a una forma di esperienza che può ben qualificarsi - come alcuni studiosi hanno opportunamente rilevato - proprio come una "esperienza mistica": la prima forma di esperienza mistica espressa a livello filosofico.

In effetti, in tutta la grandiosa pagina del finale del discorso messo in bocca alla sacerdotessa Diotima di Mantinea, e soprattutto nelle espressioni «E questo il momento della vita che più di ogni altro è degno di essere vissuto da un uomo, quando contempla il bello in sé», e in particolare «contemplarlo solo e stare insieme a lui» e «contemplare e rimanere unito a esso», anticipano proprio quel- 
la che sarà chiamata "esperienza mistica", in cui il soggetto si unifica e fonde con l'oggetto.

In riferimento al finale del discorso di Diotima, A.E. Taylor scriveva: «Nonostante tutte le differenze di propositi, il miglior commento all'intero racconto è fornito dai grandi scrittori che, in verso o in prosa, hanno descritto le tappe della "via mistica", mediante la quale l'anima "esce da se stessa", per trovarsi di nuovo trovando Dio. In sostanza, quello che Socrate sta descrivendo è lo stesso viaggio spirituale che descrive, per esempio, S. Giovanni della Croce nella nota canzone En una nocbe oscura, con cui si apre il suo trattato Notte Oscura, o quello a cui Crashaw accenna più oscuramente in tutte le pagine del suo The Flaming Heart, e che Bonaventura ci mostra con nitidezza nell'Itinerarium mentis in Deum. [...] Nella letteratura greca, a mio parere, questo discorso rimane senza eco fino a Plotino, con il quale la medesima avventura spirituale si ripete come tema essenziale delle Enneadi. Se non abbiamo in noi quel tanto di misticismo che è necessario per considerare l'annullarsi e il rinnovarsi dell'anima come il compito essenziale della vita, il discorso non avrà per noi un valore reale e non potremo far altro che considerarlo un "bel sogno" mitologico» (Platone, Firenze 1968, p. 351).

$\mathrm{E}$ in riferimento a quel manifestarsi del Bello in sé

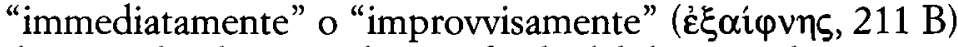
di cui parla Platone nel passo finale del discorso di Diotima, G. Krüger precisa: «Questo "lampo di una trepidante visione (ictus trepidantis aspectus)" come lo chiama Agostino, proprio nel senso di Platone, è ciò che c'è di misterioso nel "mistero" dell'Eros: l'evento dell'illuminazione in cui ogni singolo è insostituibile. A chi non ne fa esperienza di persona non può spiegarlo né Platone né un interprete". E, insieme a Taylor, Krüger su questo atto di "esperienza mistica" ribadisce: "O uno lo possiede e ne è posseduto, oppure non lo possiede, e allora non c'è altro da dire» (Ragione e passione, Milano 1995, p. 208). 


\section{Totale assenza di riferimenti alle testimonianze sulle "dottrine non scritte" di Platone e sul loro significato}

Già nel 1929, subito dopo la pubblicazione del primo volume del Platone, Adolfo Levi, in una recensione pubblicata nella «Rivista di Filologia e di istruzione classica», denunciava questo errore.

Pur essendo legato ad alcune convinzioni del tutto superate dagli studi successivi (in particolare che nella Repubblica Platone non aveva ancor pensieri chiari sul Bene e che le dottrine non scritte risalgono alla ultima fase del pensiero del filosofo) e prima ancora che la questione delle dottrine non scritte emergesse in primo piano, Levi faceva giusti rilievi che mette conto rileggere: «[...] l'ultima espressione del pensiero platonico, la teoria delle Idee numeri e dei Numeri Ideali (di cui assai arbitrariamente l'Autore non tiene alcun conto), ponendo come elemento formale del mondo ideale l'Uno, concepito come equivalente all'Essere, mostra in modo chiaro come Platone cercasse di esprimere in termini esattamente razionali il supremo principio della vera realtà, superando anche in questo punto la cerchia delle convinzioni non giustificabilis (p. 537).

In effetti, circa le dottrine non scritte si possono sostenere tesi di vario genere, ma nessuno può correttamente negare che ci siano state: di conseguenza non si può non parlarne.

Inoltre, a partire almeno dal Simposio e via via in maniera più frequente nei vari dialoghi ci sono allusioni e rimandi alle dottrine non scritte sempre più forti, addirittura con allusioni assai spinte e con comunicazioni quasi scoperte dei concetti-base concernenti i Principi primi e supremi, fino alla esplicita affermazione nel Filebo che identifica il valore supremo con la "Misura" (il Bene è l'Uno, Misura di tutte le cose).

Inoltre, in vari dialoghi si riscontrano accentuati riflessi della funzione e della dinamica dei due Principi primi del Bene come Uno e del Male come divisione e molteplicità disordinata. In effetti, la struttura bipolare del reale emerge da un capo all'altro degli scritti platonici, con al vertice Par- 
menide e il Filebo, come il lettore interessato potrà vedere, con la relativa documentazione, nella nostra opera Per una nuova interpretazione di Platone.

$\mathrm{Ma}$ il silenzio su tali dottrine dipende da quella "chiusura ermeneutica" imposta dal paradigma interpretativo seguito da Friedländer, che, se fa vedere alcune cose molto bene, non ne lascia vedere altre.

In che senso tale "chiusura paradigmatica" risulti determinante si può ricavare anche dal modo in cui lo studioso mostra di non aver compreso il messaggio dell'opera di Hans Krämer, Arete bei Platon und Aristoteles (1959), la quale presenta un nuovo paradigma ermeneutico alternativo, e che viene espressamente citata (cfr. infra, p. 158, n. 19; 168, n. $3 ; 204$, n. 5 ). Il lettore interessato può vedere H. Krämer, Platone e $i$ fondamenti della metafisica, Milano 1987; 20016, opera da noi tradotta e introdotta.

\section{La cifra spirituale emblematica del "Platone" di Friedländer}

Degli assi portanti dell'opera di Friedländer abbiamo parlato sopra diffusamente, ma per concludere vorremmo richiamare quei concetti che - a nostro avviso - rivelano la cifra emblematica dell'intera opera.

Lidea di fondo che rende unitario il discorso di Friedländer su Platone ci sembra questo: «Kosmos è la compagine così del mondo, come dello Stato e come dell'anima» (infra, p. 42), con il richiamo di Gorgia, 507 E sgg.

Si tratta della struttura ontologica e metafisica simmetrica sussistente fra l'Universo, la Polis e l'Anima, che rappresenta nel modo più elevato e più perfetto una costante ricerca dei Greci di spiegare come l'uomo possa essere felice, collocandosi nel cosmo in giusta misura e in armonia.

Lo studioso precisa: «appena si getta uno sguardo sul Timeo, il quale, ancora una volta, raffigura l'immagine dell'edificio statale, prima di erigere l'edificio cosmico, ecco che la simmetria di stato e cosmo diventa lampante. Quando 
nel Timeo viene detto (90 C D) che compito dell'uomo è imparare le armonie e i moti circolari dell'universo e assimilare quel che è da conoscere a ciò che è conosciuto secondo

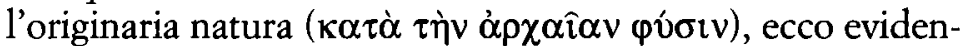
te la simmetria fra anima del singolo e cosmo. E sull'omologia di anima e stato riposa l'intera costruzione della Repubblica platonica. E significa davvero leggerla nel senso di Platone, quando nel mito finale si veda completarsi il nesso d'insieme: anima umana, stato, cosmo, concepiti come tre forme collocate in simmetria intorno allo stesso centro; e tuttavia non come sfere separate, per quanto tra di loro di ugual forma; ma come l'uomo, secondo la sua propria natura, appartiene allo stato, così sembra appartenere, secondo la sua propria natura, anche al cosmo. Come nel Fedone la sfera terrestre, così qui l'edificio del mondo appare costruito per procurare alle anime umane lo spazio adeguato a ciascuna di esse» (infra, p. 217).

Ma Platone ha fatto questo solo in conseguenza dell'incontro con Socrate, che fu il suo "destino". In Platone è Socrate che rivive e parla: «Prova dell'anima e guida alla virtù e alla polis, è il dialogo d'amore per Socrate e Platone, i quali sono profondamente concordi al riguardo e da distinguersi meno che per qualsiasi altra questione. E una generale legge giunge alla più evidente forma nel Socrate platonico, per la quale ci si può richiamare alle sentenze di tre grandissimi: di Hölderlin: "Il meglio che il mortale può dare lo dà amando"; di Goethe: "In generale si impara soltanto da chi si ama"; di Nietzsche: "Soltanto dall'amore nascono le più profonde intuizioni". Questi detti si integrano in modo mirabile e Ernst Bertram nel suo libro su Nietzsche li ha ridotti a un accordo a tre: "Hölderlin si riferisce a chi insegna, Goethe a chi impara, Nietzsche a quel che nasce dalla loro unione". Tutti e tre questi elementi ritroviamo in Platone, e ora del terzo dobbiamo ancora parlare. Eros fa incontrare due persone e quando queste persone si chiamano Socrate e Alcibiade esse devono filosofare l'una con l'altra. Questo hanno visto gli altri. Ma Platone vede di più. $\mathrm{Ha}$ visto la forza di questo grande demone allargarsi in una 
nuova dimensione: non solo l'amatore insegna e l'amato impara, ma è appunto da questo amore che "nascono le più profonde intuizioni". Così esso diviene guida all'idea, ed è questa in primo luogo la svolta propriamente platonica. Anch'essa appare sempre come una interpretazione della figura di Socrate, ma conduce a una profondità di fronte alla quale il Socrate storico sarebbe forse rimasto meravigliato» (infra, pp. 66 sg.).

Friedländer sarebbe favorevole al ricupero dell'autenticità della Lettera II, e ritiene che, comunque, alcune cose in essa contenute si impongano come delle verità incontrovertibili, in particolare in quelle parole in cui l'Autore della Lettera II caratterizza i dialoghi platonici come «pensieri di Socrate quando era bello e giovane» $(314 \mathrm{C})$.

$\grave{E}$ una affermazione, questa, che esprime in maniera perfetta l'interpretazione stessa di Friedländer, il cui Platone è appunto un Socrate giovane e bello, che ha visto nel Socrate vecchio la verità e l'ha espressa, esplicitandola, approfondendola e completandola.

A proposito del passo della Lettera II, lo studioso scrive: $\ll \mathrm{E}$ anche se queste parole non dovesse averle scritte Platone, come molti ancora oggi pensano, tuttavia rimarrebbero sempre la più forte e, nella loro fantasticheria, la più propria espressione di ciò che anche per noi è il tratto peculiare dei suoi scritti» (infra, p. 157). 
